\title{
Investigation into Challenges Faced by Employees in Implementing a Safety Strategy in a South African Platinum Mine
}

\author{
Maphsuka Tibane ${ }^{1}$, L. Niemand ${ }^{2}$ \\ ${ }^{1}$ Department of Business Studies Management College of Southern Africa (MANCOSA) \\ ${ }^{2}$ Department of Business Studies Management College of Southern Africa (MANCOSA)
}

\begin{abstract}
Since the early part of the 19th century, the mining industry has been a significant source of employment and major contributor to Gross Domestic Product (GDP) in South Africa. However, mining is also one of the most hazardous and dangerous occupations. The new democratic dispensation in South Africa saw major improvements in the regulation and approach to safety in mining. Despite the increased focus on safety, the mining industry in South Africa continues to be plagued by deaths relating to poor safety. Although the mine's safety strategy, procedures and systems are wide-ranging in principle, it appears that adherence to the same is not optimal. It was therefore important to understand the challenges experienced by employees regarding safety compliance in order to mitigate these challenges and strive towards the envisaged Zero Harm initiative. The purpose of this study was to investigate the challenges faced by employees in complying with the safety standards at the South African mine. A quantitative, descriptive research approach was used to collect responses from 116 participants. The findings from the descriptive quantitative analysis reveal that there is a relatively high degree of awareness of the safety strategy and safety system. However, challenges related to the complexity of the safety system and commitment from management towards employee safety was uncovered. Based on the primary findings, the recommendations focused on three important areas, namely; communication, employee training and management's involvement in order to improve the implementation of the safety strategy and safety system at the mine.
\end{abstract}

Keywords: mine safety, safety compliance, management commitment to safety standards

\section{INTRODUCTION}

Prior to 1993, employees in South Africa's mining sector suffered an average of 800 deaths and experienced in excess of 18000 injuries per annum. However, with the establishment of the Mine Health and Safety Council (MHSC), the increased regulation through the promulgation of the Mine Health and Safety Act (MHSA) and a more intensive drive to improve safety from all stakeholders in the sector, there has been a major improvement in terms of mine fatalities and injuries. The number of fatalities resulting from poor safety reduced drastically from more than 600 in 1993 to 84 in 2014 . Fatalities resulting from poor safety in the platinum mining sector accounted for approximately $18 \%$ of all recorded fatalities in 2014 (Mine Health and Safety Council, 2015:3). The mine, like all other major companies in the mining sector, has strived towards a Zero Harm initiative wherein there is a concerted drive and substantial investments towards ensuring that every mine worker returns home unharmed every day. The mine's commitment towards employee safety is entrenched in its value statements, where it is stated that; "We care for the health and safety of all our stakeholders". Supporting the pursuit of this value and giving credence to the statement that the mine cares for the health and safety of all its stakeholders is a comprehensive safety strategy.

\section{Problem Statement}

Safety compliance is defined by Masia and Pienaar (2011:212) as the extent to which safety standards, legal obligations and procedures are adhered to by employees. Non-compliance to safety procedures and failing to uphold a culture of safety will inevitably lead to injuries and fatalities for employees and stoppages and poor public relations for employers. Safety in the mining industry has always been an area of concern.

\section{Aim of the Study}

The aim of this study is to investigate the challenges faced by employees in implementing the safety strategy and complying with the safety system of the South African mine. 
Maphsuka Tibane L.\& Niemand "Investigation into Challenges Faced by Employees in Implementing a Safety Strategy in a South African Platinum Mine"

\section{Research Questions}

The research questions are as follow:

- To what extent are employees aware of the safety strategy and system at the mine?

- What are the challenges experienced by employees in implementing the safety strategy and adhering to safety system at the mine?

- How can management improve the implementation of the safety strategy as well as adherence to safety standards and procedures amongst employees at the mine?

\section{Significance of the Study}

It can be easily argued that any research study which seeks to mitigate risks that lead to the loss of human life is significant. Research relating to health and safety in the workplace is widely available across many industries for the simple reason that all stakeholders have a vested interest in protecting human life. Research relating to safety in the mining industry, locally and globally, is conducted by businesses as well as academics who study the field. In most cases, the significance of each study is attributed to the devastating effects that poor safety can lead to. For example, the victims of fatal mining accidents resulting from poor safety are often the breadwinners of their household. This impact of safety related fatalities thus extends far beyond the immediate victims to their families. From a business perspective, besides the loss of an employee, operations are often shut down following a safety related fatality. The output loss incurred during these shut downs place the profitability of the companies at risk. Fines and penalties issued for poor safety conditions also impact the profitability of mining companies. As such, safety is a very important business concern of mines and any research that seeks to identify weaknesses in safety as well as proactive measures to enhance safety is considered significant by all stakeholders like management, owners and employees in the mining industry.

\section{LITERATURE REVIEW}

The role of the South African mining industry over the past decade cannot be underestimated. Today, the mining industry contributes approximately six per cent to the Gross Domestic Product of South Africa and it has been a major driver of the emergence of the country as a regional and continental powerhouse. Growth in the industry has been phenomenal and today, South Africa's mines extract more than three quarters of the world's platinum. However, since the discovery of precious metals such as gold and platinum in South Africa, the mining industry has been plagued by workers' injuries and fatalities resulting from unsafe conditions in spite of a combination of legislation and commitment from mining stakeholders. Historically, transport accidents, as well as falls of ground, have been the major safety related causes of injuries and fatalities amongst employees. However, in recent times, there has been an increase in safety related injuries and fatalities occurring from explosives use, machinery use and general operations within the mines (Mine Health and Safety Council, 2014:2). According to Masia and Pienaar (2011:212), the term safety compliance is used to refer to the extent to which employees adhere to safety requirements, safety procedures, safety standards and safety legislation.

\section{Mine Safety Legislation and Regulations in South Africa}

Prior to 1994, safety performance of mining companies was appalling. The number of fatalities and injuries suffered by employees due to unsafe conditions were almost ten times worse than what they are today. A major catalyst and contributor to the substantial decrease in injuries and fatalities was the list of recommendations put forth by the Leon Commission of Inquiry into health and safety at mines. The inquiry was commissioned by late and former President Nelson Mandela as an attempt to find a solution to the devastating effects caused by unsafe conditions at mines in South Africa. The commission was to investigate the relevance and adequacy of existing legislation and to make recommendations based on prevailing international best practices oriented towards the safety and health of employees in mining. The inquiry found the existing legislation to be vastly inappropriate and in the months following the inquiry's report, the findings were used to promulgate the Mine Health and Safety Act (MHSA), Act 29 of 1996 (Chamber of Mines of South Africa, 2016:8). In attempting to address the shortfalls of the previous safety legislations, the MHSA (Act 29 of 1996) and subsequent amendments (1996 and 2006) seeks to protect the safety of employees by: 
Maphsuka Tibane L.\& Niemand "Investigation into Challenges Faced by Employees in Implementing a Safety Strategy in a South African Platinum Mine"

- Making it compulsory for employees to participate in decision making related to employee safety, through the provision of safety representatives and safety committees

- Requiring both the employer and employee to work together in identifying safety hazards and thereafter adopting measures to control, minimise and eliminate these hazards

- Making it a compulsory activity for mining firms to put measures in place to effectively monitor safety conditions at mines and thereafter enforce these measures rigorously

- Requiring that mining companies conduct investigations and inquiries that are oriented towards improving safety at mines

- Developing a safety culture amongst employees as well as providing for the necessary training requirements of employees.

- Creating a framework for consultation and engagement between all stakeholders (the Government, Unions, Employers and Employees) with the view of enhancing the safety performance of the mining industry (Zungu, 2016:13)

\section{Theory of Accident Causation}

In order to understand the driving force of safety systems theory, one needs to understand the primary reason for safety systems being implemented in the first instance. According to Katsakiori, Sakellaropoulos and Manatakis (2009:1008) accidents are the primary reason why approaches, systems and strategies have been adopted to ensure safety of employees. Understanding how accidents occur is also very important to comprehend how safety systems are created. Therefore, understanding how accidents occur lies at the foundation of all accident prevention and safety system efforts. Traditionally, accidents have been viewed as resulting from a chain of events, each directly related to its "causal" event/s. The event/s at the beginning of the chain is labelled the root cause (Katsakiori et al., 2009:1008). Accident causation models were originally developed in order to assist people who had to investigate occupational accidents, so that such accidents could be investigated effectively. Knowing how accidents are caused is also useful in a proactive sense in order to identify what types of failures or errors generally cause accidents, and so action can be taken to address these failures before they have the chance to occur (Saleh, Marais, Bakolas, and Cowlagi, 2010:1119). The Domino Theory below will explain where the injuries emanate from.

\section{The Domino Theory}

In 1931, H. Heinrich presented a set of theorems known as "the axioms of industrial safety". The first axiom dealt with accident causation, stating that 'the occurrence of an injury invariably results from a complicated sequence of factors, the last one of which being the accident itself.' A model known as the 'domino theory' was then also presented by Heinrich (cited in Sabet, Aadal, Jamshidi and Rad, 2013:74) as this accident sequence was likened to a row of dominoes knocking each other down in a row. The sequence is: Injury, caused by an; Accident, due to an; Unsafe act and/or mechanical or physical hazard, due to the; Fault of the Person, caused by their; Ancestry and Social Environment (Sabet et al., 2013:74). The accident is avoided, according to Heinrich (cited in Sabet $e t$ al., 2013:74) by removing one of the dominoes, normally the middle one or unsafe act. This theory provided the foundation for accident prevention measures aimed at preventing unsafe acts or unsafe conditions. However, the domino model has been noted as a one-dimensional sequence of events (Sabet et al., 2013:74). Hosseinian and Torghabeh (2012:55) argue that accidents are usually multifactoral and develop through relatively lengthy sequences of changes and errors. This has led to the principle of multiple causation, which is discussed below.

\section{Principle of Multiple Causation}

According to Hamid, Majid and Singh (2008:243), behind every accident there lies many contributing factors, causes and sub-causes. The theory of multiple causation is that these factors combine together, in random fashion, causing accidents. So, during accident investigations, there is a need to identify as many of these causes as possible, rather than just one for each stage of the domino sequence. The accident model is in reality an amalgam of both the domino and multi-causality theories. The review of accident causation theory points to a common conclusion. All accidents whether major or minor are caused, there is no such thing as an accidental accident. Therefore, it can 
Maphsuka Tibane L.\& Niemand "Investigation into Challenges Faced by Employees in Implementing a Safety Strategy in a South African Platinum Mine"

be said that accidents, particularly in large organisations and dangerous working environments are seldom associated with a single cause. The causes of accidents are usually complex and interactive. Based on this assumption, in the following subsection systems theory will be explained. A systemstheoretical approach to understanding accident causation allows more complex relationships between events to be considered and also provides a way to look more deeply at why an accident occurred. This, in turn, supports the effective creation and progress of safety systems.

\section{Systems Theory and Safety}

Accident causation theory is largely based a post-incident activity and there has been a growing need to conduct research in a proactive manner so as to reduce accidents and improve the safety of employees (Weick, 2004:28). System theory attempts to address the issue of proactive research and this theory postulates that accidents are a result of flawed processes. In this regard, if a system is optimised, safety performance will improve through fewer accidents. By viewing a system as a group of interrelated components which include; people, practices, policies, procedures and machinery, a non-linear and dynamic model emerges. In an occupational safety system, besides employees, policies and procedures, integrated components include, safety committees, organisational structures that promote accountability, interactions with external stakeholders such as regulators, monitoring and evaluating safety conditions, leadership and feedback channels (Lei and Yuanyuan, 2014:202). This model, unlike the accident causation theory, is less likely to place blame purely on employees without understanding the factors that that influence employees to engage in unsafe acts.

\section{Typical Elements of a Safety System}

Safety systems are a combination of various elements or components. The composition of a safety system is determined by the type of hazardous activities being conducted, the nature of the product being excavated, the equipment being used, the hazard and risk associated with the environment and a plethora of other important factors (Holt, 2008:15). However, Saurin, Formoso and Cambrai (2008:1181) found that there are core elements that support effective safety systems. Firstly, a safety policy and commitment by the management on which the system is based is critical in terms of forming a base upon which the safety system is to be implemented. However, an effective safety policy is heavily reliant on three processes, namely; a process for identifying hazards to safety and for evaluating and managing the associated risks; a process for ensuring that personnel are trained and competent to perform their duties and a process for the internal reporting and analysing of hazards, incidents and accidents and for taking corrective actions to prevent their recurrence. Setting goals for the improvement of safety and for measuring the attainment of those goals is thereafter another critical element of an effective safety system. Other typical elements of an effective safety system include; a document containing all safety management system processes and a process for making personnel aware of their responsibilities with respect to them; a quality assurance programme and a process for conducting periodic reviews or audits of the safety management system (Saurin et al., 2008:1181). In addition to the typical elements of a safety system, Priemus and Ale (2010:116), deduced that the following guiding principles were necessary in order for safety systems to be effective:

Management Commitment to Safety: Management commitment to safety is important because the attitudes and actions of management can significantly influence the culture of safety in an organisation and it is therefore critical that leaders commit to the success of the implementation and continuous monitoring of the safety system (Priemus and Ale 2010:117).

Proactive Identification of Hazards: Early identification and reporting of hazards is regarded as critical to the monitoring and improvement of any safety system (Priemus and Ale 2010:117).

Actions Takent Manage Risks: A process must be in place to determine logical approaches to counteract known risks to safe operation (Priemus and Ale 2010:117).

Evaluation of Safety Actions: An ongoing evaluation of the impacts of risk management actions is necessary to determine if further remedial activities are required (Priemus and Ale 2010:117).

\section{Implementation of Safety Systems}

The essence of understanding safety systems is not just in defining it and identifying its components or elements but also in effectively implementing it. In all mining companies globally, safety is interpreted in bold letters. The importance of safety systems in mining is emphasized by a coordinated 
Maphsuka Tibane L.\& Niemand "Investigation into Challenges Faced by Employees in Implementing a Safety Strategy in a South African Platinum Mine"

approach between safety stakeholders in a multipronged effort. Like with any company strategy or system, the implementation of safety systems in mining does come with its own problems and limitations. As the reliability and capability of technology improves, it is generally accepted that most mining accidents result due to human error. It would be easy to conclude that these errors indicate carelessness or incompetence on the job, but that would not be accurate (Aksorn and Hadikusumo, 2008:711). Carbonari, Giretti and Naticchia (2011:689) argue that the human factor is the weakest link in a chain that leads to an accident. These accidents can be prevented by not merely changing people but also by adopting well defined safety systems and implementing these successfully.

\section{Approaches to Safety Systems Implementation}

Safety system implementation has posed a very big challenge for most mining companies in the world. Wang and Wang (2010:1115) found that a range of implementation approaches have been adopted. The variety of implementation approaches is due in part to the extent of customization required to suit the size and nature of mining operations within different settings. Although safety is not a new concept in the mining industry, the issue of safety system implementation is. Despite this, larger mining firms have found success in adopting one of the following three approaches to safety system implementation (Bandyopadhyay, Chaulya, Mishra, Choure and Baveja, 2009:119).

Evolutionary Style: In this approach, the mining company implement safety systems principles over a period of several years. Over the course of this time, the safety culture gradually becomes ingrained in employees' attitudes and actions by means of their experience and work culture. Every employee is briefed again and again by the safety teams dedicated to create safety awareness among them (Bandyopadhyay et al., 2009:119).

Phased Methodology: This approach uses dates and milestones to implement the various aspects of a safety strategy. It allows time to address any issues that arise before advancing to the next stage. However, this approach can only be successfully adopted if some elements of the safety strategy can be confidently given priority over others. Often, the elements of a safety system are all equally important and management cannot delay in implementing them at different phases (Bandyopadhyay et al., 2009:119).

Fast Track Adoption: This approach seeks to implement the safety system at a relatively rapid pace. Although this approach may bring the organisation into compliance with safety regulations, it may not result in a sufficiently fundamental change in safety practices, attitudes and the general culture of safety. In some instances, an aggressive employee training program will be required to achieve this sort of quick transition. However, the fast track adoption approach has seldom been sustainable in the long-term (Bandyopadhyay et al., 2009:119). Wang and Wang (2010:1117) found that when implementing a safety system, organisations who focused on three aspects, appeared to benefit from greater implementation success:

Document Control: Documentation is a key area to record any change in procedures. It allows employees to be updated and knowledgeable about what is going on. Therefore, proper documentation is key to enhancing safety system implementation processes (Wang and Wang, 2010:1117).

Audits by Self and Third Party: It is essential to audit, review and revalidate procedures and all methodologies on a regular basis to ensure that they are current and effective. The self-audit is necessary in order to force employees to reflect on the safety of all actions undertaken. Third party audits are necessary for the purposes of objectivity and identifying "bad habits" which compromise safety (Wang and Wang, 2010:1117).

Safety System Training at Executive Level: It is perhaps imprudent to assume that executives will automatically understand the importance of safety systems. Executive Management need to be well briefed about the importance and consequences of an effective safety system in the organisation. In this regard, training for executives relating to all aspects of the safety system implementation is crucial (Wang and Wang, 2010:1118). Safety receives considerable attention and investment at the mine. During 2015, the mine invested more than two-hundred million Rand in activities and solutions aimed at executing its safety strategy. The safety strategy is based on comprehensive reviews (external and internal) of the company's safety system. Fostering a culture of safety with the view of achieving the target of Zero Harm "every worker returning home safe every day" is the core thrust of the safety strategy at the mine. The focus of the safety strategy is also an echo of the major values of 
Maphsuka Tibane L.\& Niemand "Investigation into Challenges Faced by Employees in Implementing a Safety Strategy in a South African Platinum Mine"

the company, which is to care for the health and safety of all its stakeholders. The safety system at the mine is crucial to the company's sustainable development creation objectives where a target of zero fatalities has been set for the 2016 financial year. The mine initiated a strategic plan as follows:

- Initiatives within the organisation that is used to promote a safety culture are guided by the Culture Transformation Framework which is an industry adopted best practice guide. The CTF is an eleven pillar framework which encourages mining companies to revolutionise approaches to employee safety through the creation of a culture that is mindful of risks and consistently seeks to improve safety at mines. In terms of effective leadership, the safety strategy implores upon the executive team to promote the envisaged safety culture and to provide visible commitment towards safety.

- Senior managers are also responsible for driving the Zero Incident Process (ZIP) programme which aims to improve safety awareness amongst employees. In addressing motivation, an incentive scheme based on meeting targets for output and safety has been implemented for certain managerial levels. The strategic pillar dealing with "Practices", prioritises the risk management system, Triggered Action Response Plan (TARP), Women in Mining (WiM), Occupational Health and Safety Assessment Series (OHSAS) 18001, Critical safe behaviours, Section 23 actions and Safety training.

- The risk management system seems to minimise the 14 risks and hazards that have been identified as impacting negatively safety through the effective implementation of relevant controls.

- The Critical safe behaviours programme is aimed at creating awareness and training employees on the correct behaviours that are required within specific occupations in order to remain safe.

- Section 22 and 23 training is also provided to ensure that employees are aware of their rights in the event of there being a danger to their safety.

- A ten-day safety programme is provided to approximately $10 \%$ of all employees every year.

- The strategic pillar dealing with "Safety Environment", prioritises the investment in machinery and other technical equipment in order to mitigate risks introduced through the human element during operations. Amongst these are; Proximity Detection Systems (PDS) on trackless Machinery, Vertical shaft bank and station safety devices, Roof bolts and nets, Electronic winch signalling devices, Fire retardant conveyor belts, Underground fire detection system enhancements and Gas measuring instruments.

A vast amount of money was spent on the systems but the improvement is not at the rate that was expected.

\section{Employee Awareness of Safety Strategies and Systems}

Implemented safety strategies and systems, regardless of how comprehensive they may be, will be ineffective if there is low compliance due to poor awareness. Mokoena and Oberholzer (2015:2) found that knowledge of legislative safety requirements and compliance to safety systems at South African mines is sorely lacking. So serious is the matter of safety at mines in South Africa that companies, government and stakeholders have come together to produce a mass of safety related legislation, policies, best-practices, systems, and lengthy strategies to encompass the same. However, like in any other corporate or business setting, the value of a strategy is not realised through the pieces of paper on which it is documented, but rather through organisational-wide awareness, buy-in and implementation (Mokoena and Oberholzer, 2015:2).

\section{Challenges Experienced by Employees with Safety Compliance}

Literature relating to poor safety compliance and causes thereof is available in the form of mining disaster, fatality and injury reports. However, to date, there is limited literature on the reasons which lead to the causes of fatalities and injuries amongst mine employees in South Africa. This viewpoint is important in developing strategies to minimise safety hazards brought upon by unsafe acts by employees. For example, the Department of Mining and Energy reports that the causes of safety hazards which lead to injuries and fatalities amongst mine employees in South Africa are falls of ground, poor incorrect use of machinery and irregular use of transportation vehicles. However, the key question that remains is; if employees know of the dangers, why do they still not comply with safety rules and systems? Zungu (2016:14) believes that the targets envisaged through the Zero Harm 
Maphsuka Tibane L.\& Niemand "Investigation into Challenges Faced by Employees in Implementing a Safety Strategy in a South African Platinum Mine"

campaign can only be realistically achieved if more is done to understand the plight of the average employee in complying with safety systems. Arguably, the production targets of most mining companies, both locally and abroad, is often the underlying cause of poor compliance to safety systems by employees. In scenarios where there has been a stoppage in production, for example; during a strike, a natural disaster or a death at a specific shaft, employees are often pushed to make up for lost production. The increased hours of operation and pressure to meet higher targets result in employees "doing things faster" often at the expense of safety compliance (Lim, Murray, Dowdeswell, Glynn and Sonnenberg, 2011:6). Other key challenges to implementing safety systems are listed and discussed below.

Top Management Support: Unless the board and senior management make a visible commitment to institutionalizing safety systems, the unsafe acts will remain the status quo. Top management support means changing the reward structure and imposing difficult consequences for those employees and volunteers who do not engage in safety practices that are in adherence with the safety system. Top management's modelling the desired behaviour is essential for a safety system to be successful (Saleh et al., 2010:1108).

Lack of Appreciation for the Safety System: Employees at all levels of the organisation need to understand why the safety system is a crucial issue and how to actively and effectively implement it. It can be difficult to convey that knowledge and appreciation to an entire organisation - especially when employees are based underground for long hours. Training is one way of beginning the process (Saleh et al., 2010:1108).

Time: Implementing a safety system is an investment of time and energy. Time, however, can act as a barrier to implementing a safety system, regardless of how well it may be conceived and presented. Time is always at a premium and therefore senior management commitment and priorities need to be set to allow the rest of the organisation to invest the necessary amount of time and energy implement and adhere to the safety system (Saleh et al., 2010:1108).

Communication: Lack of clarity about the issues surrounding safety and the consequences of accidents and injuries are often barriers to workplace safety systems. The organization needs to clearly and consistently communicate performance expectations about safety. The potential cost of accidents and injuries need to be addressed as well (Saleh et al., 2010:1108).

Recommendations to Support the Effective Implementation of Safety Systems: Although it is helpful to identify potential barriers to implementing an effective safety system, it is more important to identify ways to overcome those barriers. Management's commitment and support for safety systems may be measured or judged. More significantly, when managers are associated with the safety system, they begin to own the costs of injuries and accidents within their departments. By linking performance reviews and financial incentives with safety goals and objectives, employees and managers can observe senior management's commitment to change. Safety can also be a conduit that shows the link between efficiency and employee or volunteer morale. Emphasis on safety shows employees and volunteers that the organization cares about their well-being, and can open the door to better management/employee/volunteer relations. Management also achieves the same objectives by linking safety to the organisation's mission and demonstrating how commitment to safety is commitment to the organisation's mission (Green, Bosscha, Candy, Hlophe, Coetzee and Brink 2010:13).

\section{Conclusion}

From the available literature which reports on the challenges experienced by workers with safety compliance in the mining industry, it evident that research in this areas is relatively underdeveloped. Therefore, this research study seeks to add to the body of literature which deals with the challenges relating to awareness and compliance with safety systems. Given the nature of mining operations at the investigation site (the findings will most probably be generalisable to other mining companies with similar resources and operating in similar conditions.

\section{RESEARCH METHODOLOGY}

A research philosophy is a holistic view about the thinking and practices that ultimately guide various research techniques (Zikmund, Babin, Carr and Griffen, 2012:60). A research philosophy is also often referred to as the conceptual framework that is adopted by a researcher when attempting to discover 
Maphsuka Tibane L.\& Niemand "Investigation into Challenges Faced by Employees in Implementing a Safety Strategy in a South African Platinum Mine"

solutions through the examination of a defined problem. Although the term research philosophy may seem abstract, it is often made visible through the structures, patterns, approaches that are adopted by researchers when conducting research (Collins and Hussy, 2013:114). Two major research philosophies are positivism and phenomenology (Burns and Burns, 2008:93). Positivism is based on the premise that reality can be objectively documented via experiment and observation. Quantitative measures are adopted in positivism in order to describe quantifiable parameters. Researchers who adopt the positivism philosophy believe that knowledge is generated through facts since reality is documented via quantitative measures. Objective measures, parameters and reporting indices are given critical importance under the positivism philosophy (Bryman and Bell, 2015:58). Therefore, a key tenet of the positivism philosophy is that knowledge is created through description of events instead of questioning the reason for the event and how the event occurred. It can also be said that positivist researchers need to see and count facts in order to measure observable events. In this regard, beliefs and subjective thoughts are not valued in positivism since they are regarded as unscientific. As such, positivism on structures research designs and research approaches in order to generate findings that are generalizable and reproducible. For the purposes of this study, the positivism philosophy is adopted since it is important to quantify parameters related to safety. Positivism also guided the researcher in adopting a quantitative research approach, which is regarded as being necessary in order to obtain objective measures of variables as well as ensure representation of the target population.

\section{The Research Design}

A research design is the framework and overarching structure that is used to guide the research process. A research design is also commonly referred to as the "grand plan" or the "blueprint" of the inquiry into a particular event or phenomenon. According to Cooper and Schindler (2013:9), the research context, the problem being investigated and the questions that the research seeks to answer are important considerations in the selection of the research design. Bryman and Bell, (2015:42) assert that research design guides researchers in selecting the most appropriate research approach and the most relevant research strategy. The research attempts to measure, quantify, identify and describe the variables associated with the phenomenon being investigated. Therefore, the descriptive research design was reasoned to be most appropriate for the purposes of this study.

\section{Research Approach}

A research approach is defined as the procedures that need to be conducted in order to execute research methodologically. Research approaches are guided by research philosophies and cover important aspects of scientific enquiry including; the collection, analysis and interpretation of various forms of data (Burns and Burns, 2008:109). There are two broadly defined approaches to research, namely; the quantitative and the qualitative research approaches. The quantitative research approach, as the name implies, is based on quantification and measurement. The quantitative approach is usually adopted when the study attempts to address "who, what, when, where, how many and to what extent?" research questions. Therefore the quantitative approach is reliant on validation of testing and accurate measurement. Quantitative research is often suited to descriptive research requirements which are conducted at a single point in time and which attempts to provide a generalised snapshot of a situation. The disadvantage of adopting a quantitative research approach is that it fails to provide an in-depth understanding of why and how certain events or phenomena occur (Bryman and Bell, 2015:77). Qualitative research, on the other hand, attempts to produce an in-depth explanation of a particular phenomenon, thus often requiring and understanding of elements which cannot be understood through cross sectional studies, such as emotions and human behaviour.

\section{Target Population}

In research terms, a target population comprises all elements about which or from which information is required. Target populations are not always people and can include anything from financial records to product testing data. A researcher must select the target population based on the elements' ability to provide the required data that will assist in addressing the main research questions (Kothari, 2014:94). In business research, Burns and Burn (2008:133) define a target population as all stakeholders, documents and other collections of data that are of interest to the researcher. The target population for this study was category two to eight (unskilled and semi-skilled) employees. The reason for selecting these elements as the target population is due to there being a relatively higher percentage of incidents and accident being recorded amongst employees of these categories. Employees in categories two to 
Maphsuka Tibane L.\& Niemand "Investigation into Challenges Faced by Employees in Implementing a Safety Strategy in a South African Platinum Mine"

eight are also exposed to higher levels of safety hazards due to their job requirements. The total number of employees classified within categories two to eight amounts to 150 at the specified research site.

\section{Sampling}

A sample is defined as a segment of a predefined population. Extracting a sample of the population is not always necessary. In instances where a researcher has sufficient money and time to engage with the entire target population, a universe study is conducted. However, a universe study is not always practical or feasible due to time and budgetary constraints faced by the researcher (Cooper and Schindler, 2013:113). In this study, although it would have been possible, it would have been impractical to survey all 150 of the elements in the target population. Therefore, a representative sample was selected to participate in the study.

Sampling Technique: In selecting the 116 samples from the target population, two kinds of sampling techniques were considered; probability and non-probability (Zikmund et al., 2012:68). The latter (non-probability) is generally used for qualitative research or in the absence of an adequate sampling frame. Since this study is quantitative in nature, there is an emphasis on generalisability and there is a readily available sampling frame, the study adopted a probability sampling technique. In probability sampling, every element of the population has a known and equal chance of being selected to participate in the study (Zikmund et al., 2012:68).

\section{The Research Instrument}

Quantitative data can be collected through a variety of instruments. Depending on the problem being investigated and the nature of data required, researchers generally use experiments, observations or questionnaires (Collins and Hussey, 2013:225). For this study, a survey research strategy was adopted and therefore a questionnaire was used to collect quantitative data from participants.

\section{Pilot Study}

When conducting research, researchers should conduct a pilot study before the main study. Pilot studies are often referred to as "mini version studies" or "preliminary studies" and it has been ascertained that pilot studies assist researchers in identifying flaws in their research instrument and research strategy (Zikmund et al., 2012:309). For this research, a pilot study was conducted with 10 elements of the target population. These elements were thereafter excluded from the sample selection for the main study, as per the recommendation of Kothari (2014:205). The pilot study ascertained that the instrument was reliable.

\section{Data Analysis}

There are two broad approaches to statistical analysis; namely, descriptive and inferential statistics (Burns and Burns, 2008:209). A simple difference between the two types of statistical approaches is that descriptive statistics describe the characteristics of the sample, whereas, inferential statistics tests seek to infer the findings from the sample to the population. Descriptive statistics refer to measurements which seek to describe the data set that has been compiled through data collection. These measures include central tendency measures such as mean, median and mode as well as descriptions related to the parameters (minimum and maximum values) and dispersion (interquartile range) of the data set. Inferential statistics, on the other hand, refers to statistical tests based on sample data which attempt to make inferences to the target population (Zikmund et al., 2012:166). In selecting the appropriate statistical tests, two important aspects were considered. Firstly, the study has adopted a descriptive research design. Secondly, the sample that was selected, was selected randomly at a significance level of 0.05 . This suggests that the sample is representative of the target population.

\section{Validity and Reliability}

Reliability and validity are important concepts and constructs in scientific research. Validity ensures that the research instrument measures what it claims to measure, whilst reliability estimates the extent to which the research instrument yields similar results over multiple measures or testing (Blumberg et al., 2014:155).

\section{Reliability}

In the absence of reliable measures, it would be impossible for researchers and investigators to draw sound conclusions based on findings. This would mean that theories, models and forecasts would be 
Maphsuka Tibane L.\& Niemand "Investigation into Challenges Faced by Employees in Implementing a Safety Strategy in a South African Platinum Mine"

impossible to create. Therefore reliability is a very important concept in research. Inter-rater and testretest measures of reliability were also not feasible for this study since the researcher did not have another rater or coder to assist, nor was there sufficient time to collect data twice during the pilot study. To ascertain the internal consistency of the research instrument, Cronbach's Alpha co-efficient was computed. Cronbach's Alpha co-efficient ranges from 0 to 1 , where 0 indicated no internal consistency, or reliability, and 1 indicates high internal consistency, and reliability (Collins \& Hussey, 2013:226). In most subject disciplines, including business and economics, a Cronbach Alpha coefficient of 0.7 or higher qualifies an instrument as being reliable (Bryman and Bell, 2015:223). The Cronbach Alpha co-efficient, based on the ten responses in the pilot study, showed a high level of internal consistency (0.87). Therefore, the draft research instrument was accepted for use in the main study without any amendments.

\section{Validity}

Without validity, research findings would not represent the intentions of the researcher. Validity can be divided and addressed according to two broad areas, namely; internal and external validity. External validity refers to the range in which the results of a research study are transferable or generalizable to other contexts (Kothari, 2014: 253). External validity was achieved by ensuring that the research findings can be generalised to other similar settings. This was achieved through consideration of the representation of the sample to the target population and the sampling technique.

\section{Limitations and Delimitations of the Study}

The terms limitations and delimitations are often used interchangeably in research. Although closely related, there is an elementary difference between the two terms. Limitations refer to restrictions in research that prevent researchers from obtaining extremely accurate findings. The researcher does not have control over limitations like generalizable findings and honesty. Delimitations share the same definition as limitations; however, delimitations are within the control of the researcher and are knowingly imposed often due to time and monetary constraints (Bryman and Bell, 2015:236). Although safety is a concern for all employees in a mining setting, this study was limited to level 2 to 8 staff. The reason for this delimitation in the study was based on two considerations. Firstly, injuries and fatalities are relatively higher amongst these levels as compared with any other level. Secondly, it was not feasible for the researcher to include and select a representative sample of all staff employed at the mine. Therefore, this study does not claim to be representative of the safety issues relating to every employee of at the mine.

\section{Elimination of Bias}

Being able to identify factors that create bias and thereafter eliminate or reduce the impact of these factors may significantly improve the extent to which objective, fair and critical insights are derived from a researcher's investigation. In this study, bias was eliminated by; ensuring that the questionnaire and final reported document contained gender neutral words, not identifying any of the participants by their racial or social class since it was unnecessary for the purposes of the study, avoiding phrases and narratives that suggested or reinforced any stereotypes and not making assumptions about any age groups. The use of a highly structured methodology, combined with the adoption of computer assisted data analysis also reduced the extent to which researcher bias influenced the study. The transparent presentation of all research findings through charts and tables as well as objective interpretations of the findings equally served to reduce researcher bias (Kothari, 2014:207).

\section{Ethical Considerations}

Maintaining ethical standards when conducting research is extremely important (Burns and Burns, 2008:396). The key aspects relating to ethics in research which were considered by the researcher are as follows:

- The informed consent from participants by making them aware of the title, aim, objectives and overall intention of the study using a cover sheet.

- No harm coming to the participants by providing anonymity and surveys were completed at a safe location at the work site.

- Permission was obtained. 
Maphsuka Tibane L.\& Niemand "Investigation into Challenges Faced by Employees in Implementing a Safety Strategy in a South African Platinum Mine"

- Confidentiality and anonymity was achieved by designing the questionnaire in a manner so as not to collect participant's information that could be used to identify them through their responses.

\section{RESULTS}

All 116 subjects that were selected to participate in the research, favourably responded to the invitation and completed the questionnaires. Therefore, the response rate is calculated to be $100 \%$. Relatively large portions of the sample consisted of participants who are between the ages of 30 to 39 (41\%), 40 to 49 (26\%) and 20 to $29(17 \%)$. The remaining participants are aged between 50 and 59 (15\%) and only $1 \%$ of the sample was 60 or older. The majority of participants are male $(90 \%)$ and only a small percentage are female (10\%). This is part of the history in mining which was in the past a male dominated industry. An overwhelming majority (96\%) of participants are Black, whilst the remaining $4 \%$ of respondents are White. No Indians or Coloureds participated in the study. The sample consisted of employees who have 0 to three years of work experience (15\%), four to six years of work experience (27\%), six to nine years of work experience (16\%) and 10 or more years of work experience (42\%).Twenty-two per cent of respondents have been at the mine for between 0 and three years, $24 \%$ have been at the organisation for between four and six years whilst $15 \%$ have been at the organisation for between seven and nine years. The remaining participants, 39\%, indicated that they have been at the mine for ten or more years.

\section{Employee Awareness of the Safety Strategy and System at the Mine}

Participants were asked if they are aware that there is currently a safety strategy in place at the mine. The findings indicate that there is large-scale awareness of the existence of the safety strategy being implemented at the organisation. Close to $96 \%$ of the respondents agreed or strongly agreed with this statement. Of particular importance to the employer is the fact that $1.7 \%$ of the respondents were unsure of a safety strategy being implemented and a further $1.7 \%$ and $0.9 \%$ disagreed and strongly disagreed that they were aware of the safety strategy being in place at the mine is noted. Awareness of the safety strategy was relatively high, with approximately $96 \%$ of participants indicating that they were aware of the strategy. In this questionnaire item, however, a lesser percentage of those respondents agreed to having read the safety strategy. $91.40 \%$ of respondents agreed (48.3\%) and strongly agreed $(43.103 .4 \%$ are unsure if they have read the strategy and furthermore, $3.4 \%$ and $1.7 \%$ disagreed and strongly disagreed, respectively, that they have read the safety strategy. Further to the items which sought to understand employee's awareness of the strategy and whether they have read it, this item sought to establish if they understood the safety strategy. Here too, there is a relatively high level of understanding, especially since $93.10 \%$ (59.5\% agreed and 33.60 strongly agreed) indicated such. However, there is in excess of 5\% (2.6\% unsure, 2.6 disagree and 1.7 strongly disagree) of the participants who do not understand the strategy. The questionnaire item sought to establish if participants were made aware of the possible hazards associated with their tasks. The findings indicate that a relatively large majority (94\%) (54.3\% agreed and 39.7\% strongly agreed) have been made aware of possible hazards associated with their respective tasks. However, the findings also indicate that $2.6 \%$ and $0.9 \%$ disagree and strongly disagree, respectively, that they have been made aware of the potential hazards associated with their tasks. The remaining $2.6 \%$ of respondents who indicated that they were unsure if they have been made aware of the hazards associated with their respective tasks. 50\% of the respondents respectively, strongly agreed and agreed that they were aware of the safety system in place to mitigate the risks posed by hazards which are present in their tasks. Like with preceding items, more than $5 \%$ of the respondents (4.3\% unsure and $0.9 \%$ strongly disagreed) indicated either implicitly or explicitly that they are unaware of the safety systems that have been implemented in order to mitigate the risks posed by hazards present in their task. The majority of respondents $(94 \%)$ have had their rights in terms of safety at the mine explained to them. However, $2.6 \%$ of the participants were unsure if such rights had been explained to them. The remaining participants, 3.4\% (1.7\% disagree and 1.7\% strongly disagree) indicated that their rights in terms of safety at the mine had been explained to them. In South Africa mining companies, government and stakeholders have come together to produce a mass of safety related legislation to address mining safety (MHSC, 2014:1). However, if employees are unaware of the very legislation that has been developed to delineate and protect their rights, then it can be said that their rights to being protected may never be realised. Knowing ones' duties in terms of safety and compliance is a critical step to performing them. If one does not know their duties in terms of complying with safety, it is nonsensical to expect them to perform them. $94 \%$ of the respondent in this study $(43.10 \%$ strongly 
Maphsuka Tibane L.\& Niemand "Investigation into Challenges Faced by Employees in Implementing a Safety Strategy in a South African Platinum Mine"

agree and 50.90 agree) indicated that their respective duties in terms of safety at the mine have been explain to them. More than 5\% of the respondents indicated that they were unsure if their safety duties had been explained to them and less than one per cent $(0.90 \%)$ strongly disagreed that their duties in terms of safety at the mine were explained to them. Safety system information is made available to employees, since $53.4 \%$ of the respondents agreed and a further $42.2 \%$ strongly agreed. Of the remaining participants, $2.6 \%$ were unsure, $0.9 \%$ disagreed and a further $0.9 \%$ strongly disagreed that safety system was made available. A large majority (37.10\% strongly agree and $52.60 \%$ agree) of participants felt that they could access safety system information. Almost $8 \%$ of the respondents indicated that they were unsure if safety system information was readily available and this could indicate that there was never an attempt to access such information. The remaining participants, $1.7 \%$ and $0.9 \%$ disagreed and strongly disagreed, respectively, that safety system information could be readily accessed. $50 \%$ agreed and a further $44.8 \%$ of them strongly agreed that they were fully aware of the safety procedures which they were expected to adhere to during their day-to-day tasks. The remaining participants, $1.7 \%, 2.60 \%$ and $0.90 \%$ indicated that they were unsure, that they disagreed and strongly disagreed, respectively, that they were made aware of the safety procedures that they were expected to adhere to during their day to day tasks. Emergency stops in production due to various reasons are not uncommon in dangerous occupations such as mining. It is imperative that all employees are aware of the safety procedures to adhere to during an emergency, so as not to exacerbate the situation. Whilst many respondents (49.10\% agree and $44 \%$ strongly agree) indicated that they were aware of the safety procedures to adhere to during an emergency, there are some respondents who indicated that they were unsure (5.2\%). A further $1.7 \%$ of the respondents disagreed and indicated that they were not aware of the safety procedures to adhere to during an emergency. Building a culture of safety cannot be achieved in the short term but requires a dogged approach to constantly informing employees of the importance of safety. Approximately $94 \%$ of the respondents to this research study (51.7\% agree and $42.2 \%$ strongly agree) indicated that they were constantly made aware of the importance of the safety culture at the organisation. However, it is equally important to observe that $3.4 \%$ of the participants were unsure and a further $2.6 \%$ of the participants disagreed that the organisation constantly made them aware of the importance of the safety culture at the organisation.

Challenges Experienced by Employees in Implementing the Safety Strategy and Adhering to the Safety System the Mine

$88.8 \%$ and $87.9 \%$ of respondents indicated that the regularly report safety hazards to their safety representatives and that their safety representatives report their safety concerns to the safety committee, respectively. Only $80.2 \%$ of respondents indicated that the safety committee deals with the safety hazards which are reported to safety representatives and subsequently the committee (40.5\% agreed and 39.7 strongly agreed). This implies that not all safety hazards that are reported are being addressed. To support this claim, it can be observed that $4.3 \%$ and $2.6 \%$ of respondents disagreed and strongly disagreed with this statement, respectively. The remaining $12.9 \%$ of participants indicated that they were unsure. This could mean that there is poor communication of the hazards being addressed or that the hazards were not addressed at all. The majority of respondents $(84.5 \%)$ the safety rules are written in a language that they understand (50\% agreed and $34.5 \%$ strongly agreed). However, $8.6 \%$ of the sample that indicated that the safety rules were written in a language that they do not understand, $6 \%$ disagreed and $2.6 \%$ strongly disagreed. The remaining participants, $6.9 \%$, indicated that they were unsure if the safety rules were written in a language that they understand. $37 \%$ indicated that they sometimes fail to understand which rules from the safety system to apply (26.7\% agreed and $10.3 \%$ strongly agreed). On the other hand, $34.7 \%$ disagreed that they sometimes fail to understand which rules from the safety system to apply whilst a further $7.8 \%$ of the respondents strongly disagreed with the statement. Approximately 1 in every 5 respondents (20.7\%) indicated that they were unsure. $76.7 \%$ of the respondents indicated that incentives and bonuses are made available to employees for working safely $(52.6 \%$ agreed and $24.1 \%$ strongly agreed). On the other hand, $12.1 \%$ of respondents disagreed that incentives and bonuses are made available to employees for working safely and a further $3.4 \%$ strongly disagreed. The remaining participants, $7.8 \%$, indicated that they were unsure if incentives and bonuses are made available to employees for working safely. 36.2\% indicated that when they are angry or upset with their supervisor, manager or colleagues, they do not care to adhere to the safety system ( $24.1 \%$ agreed and $12.1 \%$ strongly agreed). On the other hand, the majority of respondents (57.8\%) indicated that they 
Maphsuka Tibane L.\& Niemand "Investigation into Challenges Faced by Employees in Implementing a Safety Strategy in a South African Platinum Mine"

did not disregard adhering to the safety system when they are angry or upset with their supervisor, manager or colleagues (34.5\% disagreed and $23.3 \%$ strongly disagreed). The remaining $6 \%$ of participants indicated that they were unsure. $48.3 \%$ of respondents indicated that their supervisor sometimes complains about how difficult the safety system is to work with (32.8\% agreed and $15.5 \%$ strongly agreed). On the other hand, $34.5 \%$ disagreed that their supervisor sometimes complains about how difficult the safety system is to work with and a further $10.3 \%$ strongly disagreed with this statement. The remaining $6.9 \%$ of participants indicated that they were unsure if their supervisor sometimes complains about how difficult the safety system is to work with. The findings presented a major flaw in the implementation of an otherwise comprehensive safety system as the majority of respondents $(70.7 \%)$ indicated that management seldom disciplines employees who do not adhere to the safety system $(48.3 \%$ agreed and $22.4 \%$ strongly agreed). A relatively small percentage (18.9\%) implied that management does discipline employees who do not adhere to the safety system $(17.2 \%$ disagreed and $1.7 \%$ strongly disagreed). The remaining $10.3 \%$ of participants indicated that they were unsure if management disciplines employees who do not adhere to the safety system. The findings of the study reveal that $88.8 \%$ of the respondents regularly report safety hazards to safety representatives (42.2\% agreed and $46.6 \%$ strongly agreed). On the other hand, $2.6 \%$ disagreed that they regularly report safety hazards to safety representatives and a further $1.7 \%$ of respondents strongly disagreed with this statement. The remaining $6.9 \%$ of participants indicated that they were unsure if they regularly report safety hazards to safety representatives and this is a difficult finding to explain.

\section{CONCLUSIONS AND RECOMMENDATIONS}

Research Objective: To identify the challenges experienced by employees in implementing the safety strategy and adhering to safety system at the mine. Approximately 9 in every 10 respondents have received training to understand the safety system. However, more than $47 \%$ of the respondents stated that there are too many safety rules to remember. In excess of $8 \%$ of the respondents stated that the safety rules were written in a language that they did not understand. More than a quarter of respondents felt that mandatory PPE was not provided freely at all times. One in every two respondents felt that the safety system made easy tasks unnecessarily complex. More than $39 \%$ of the respondents felt that the safety system is sometimes impossible to apply. Approximately a third of respondents indicated that they fail to understand some of the rules of the safety system and when to apply these. More than $15 \%$ of the respondents felt that there were no incentives or bonuses to encourage employees to work safely. More than a third of the respondents stated that when they are under pressure to meet targets, they need to disobey the safety rules and system. A similar percentage of respondents stated that they would disobey the safety rules and system when they are angry or upset. Approximately $40 \%$ of the respondents felt that they needed to work around the safety system after a stoppage in production and this was followed by every 2 in 10 respondents claiming that management does not consider adherence to the safety system to be as important as production. More than $45 \%$ of the respondents also indicated that their supervisors also complain about the difficulty in adhering to the safety system. Similarly, more than two thirds of the respondents stated that management seldom disciplines employees for unsafe acts. The majority of respondents indicated that they report safety hazards to their safety representatives and more than $87 \%$ of the respondents stated that their safety representatives report their concerns to the safety committee. However, only $80 \%$ of the respondents felt that the safety committee addressed their concerns.

Research Objective: To make recommendations to management on how to improve the implementation of the safety strategy as well as adherence to safety amongst employees at the mine. The analysis of data collected from questionnaires distributed to 116 employees suggests that employee awareness of the safety strategy and the safety system at the mine is relatively high. The majority of employees appear to have been made aware of the elements of the safety strategy and the rules encompassed within the safety system. Despite the high level of awareness amongst employees, it should be noted that in order to significantly reduce safety related injuries and fatalities, it is imperative that all employees are aware of the elements of the safety strategy and the rules and procedures encompassed in the safety system. In essence, an unsafe act committed by an employee that lacks awareness of the safety strategy and safety system invariably jeopardises the safety of themselves and their co-workers. Regarding the challenges experienced by employees in implementing the safety strategy and adhering to safety system at the mine, the findings of this study have identified that the complexity of the safety system makes it difficult to remember, understand and implement. Employee training related to the safety system and the availability of information as 
Maphsuka Tibane L.\& Niemand "Investigation into Challenges Faced by Employees in Implementing a Safety Strategy in a South African Platinum Mine"

well as mandatory PPE were also noted to be challenges for employees in regards to safety compliance but not as severe as the complexity of dealing with the system. Management commitment to safety as well as the extent to

\section{RECOMMENDATIONS}

It should be noted prior to the submission of recommendations that any recommendation put forth is based on the limited scope of the study as well as subject to factors that influenced the accuracy of findings which are beyond the control of the research and which have been appropriately acknowledged under the limitations of this study. The recommendations put forth to management centre on three important aspects, namely: communication, employee training and management involvement. Communications are essential to the effective functioning of any organisation. In the mining sector however, communicating important safety information relating to the safety strategy and safety system could influence the extent to which employees are injured or lose their lives. Regular, organisation-wide communication is therefore imperative at the mine. Communication is a shared responsibility and not purely the responsibility of management. In this regard, all employees at the organisation should have a thorough understanding of the appropriate channels that must be used in order for communication of safety related information to become effective. Employee training related specifically to the safety strategy and safety system is also imperative. Although responses indicated that the majority to participants in the study have been made aware of the safety strategy and have been trained to use the safety system, it is also important to note that a small percentage responded negatively to such statements. In this regard, it is imperative that all employees receive adequate safety training as soon as possible. Ultimately, it takes one untrained employee to commit an unknowing unsafe act in order to put his/her safety and the safety of his/her colleagues at risk. Regular safety training for employees is also important for those employees who may have been trained in the past. As the results indicate, the safety system is complex. In this regard, once-off safety training programmes for employees may not have a high impact. Finally, it is recommended that management continues to demonstrate their commitment towards safety through tangible initiatives. Management commitment is a critical factor that influences that safety culture of the organisation and in this regard, employees must feel that management at the organisation is committed to their safety as much as they are committed to creating shareholder value. It is a task of the CEO to assign health and safety duties to the rest of the management under his/her control. It is impossible to mine with a disregard of the people's health and safety being first priority according to the law therefore the CEO must ensure that there are sufficient subordinates to enforce the health and safety rules. Legal appointments will ensure that people do their work and appointments do not exempt people on higher levels from being accountable. Employee relations also play a big role to ensure that everyone does what is expected of them or corrective action will be instituted against transgressors. Since the findings do not point to any major concerns relating to awareness and challenges related to the safety strategy and system at the mine, the recommendations put forth are not based on radical changes. Rather, three important areas, namely communication, employee training and management involvement have been identified in order to assist management in improving employee awareness and mitigating challenges related to the safety strategy and system at the mine.

\section{REFERENCES}

[1] MHSC (2015). 2014 Mine Occupational Health and Safety Summit. Mine Health and Safety Council South Africa, 1, pp.1-11.

[2] Masia, U. and Pienaar, J. (2011). Unravelling safety compliance in the mining industry: Examining the role of work stress, job insecurity, satisfaction and commitment as antecedents. SA Journal of Industrial Psychology, 37(1), pp. 1 - 10.

[3] MHSC (2014). South African mining industry journey to zero harm 2003 -2013. Mine Safety and Health Council.

[4] CMSA, 2015. Safety in mining: Fact sheet (2016). Chamber of Mines South Africa.

[5] Zungu, L. I. 2016. Mine health and safety council: Guidelines for the South African small-scale mining to comply with the mine health and safety act. University of South Africa, MHSC/061/1415.

[6] Katsakiori, P., Sakellaropoulos, G. and Manatakis, E. 2009. Towards an evaluation of accident investigation methods in terms of their alignment with accident causation models. Safety Science, 47(7), pp.1007-1015. 
Maphsuka Tibane L.\& Niemand "Investigation into Challenges Faced by Employees in Implementing a Safety Strategy in a South African Platinum Mine"

[7] Saleh, J., Marais, K., Bakolas, E. and Cowlagi, R. (2010). Highlights from the literature on accident causation and system safety: Review of major ideas, recent contributions, and challenges. Reliability Engineering \& System Safety, 95, pp. 1105-1116.

[8] Sabet, P., Aadal, H., Jamshidi, M. \& Rad, K. (2013). Application of domino theory to justify and prevent accident occurrence in construction site. Iosr j. Mech. Civil Eng, 6(2), pp.72-76.

[9] Hosseinian, S. and Torghabeh, Z. (2012). Major theories of construction accident causation models: A literature review. International Journal of Advances in Engineering \& Technology, 4(2), pp. 53-66.

[10] Hamid, A., Majid, M. and Singh, B. (2008). Causes of accidents at construction sites. Malaysian Journal of Civil Engineering, 20(2), pp. 242-259.

[11] Weick, K. E. 2004. Normal accident theory as frame, link and provocation. Organisational Environment Journal, 17(1), pp. 27-31.

[12] Lei, T. and Yuanyuan, D. (2014). Simulation study of coal mine safety investment based on system dynamics. International Journal of Mining Science and Technology, 24(2), pp. 201-205.

[13] Holt, A. (2008) Principles of construction safety, New York: John Wiley \& Sons.

[14] Saurin, T. A., Formoso, C. T. and Cambraia, F. B. 2008. An analysis of construction safety best practices from a cognitive systems engineering perspective. Safety science, 46(8), pp.1169-1183.

[15] Priemus, H. and Ale, B. (2010). Construction safety: An analysis of systems failure: The case of the multifunctional Bos and Lommerplein estate, Amsterdam. Safety science, 48(2), pp.111-122.

[16] Aksorn, T. \& Hadikusumo, B. (2008) Critical success factors influencing safety program performance in Thai construction projects. Safety Science, 46, pp. 709-727.

[17] Carbonari, A., Giretti, A. and Naticchia, B. (2011). A proactive system for real-time safety management in construction sites. Automation in Construction, 20(6), pp. 686-698.

[18] Wang, C. and Wang, Z. (2010). Design and implementation of safety expert information management system of coal mine based on fault tree. Journal of Software, 5(1), pp.1114-1120.

[19] Bandyopadhyay, L., Chaulya, S., Mishra, P., Choure, A. and Baveja, B. (2009). Wireless information and safety system for mines. Journal of Scientific and Industrial research, 68(2), pp.107-117.

[20] Mokoena, M. C. and Oberholzer, M. (2015). Employees' perceptions of safety control mechanisms and production cost at a mine. Problems and Perspectives in Management, 13(4), pp.70 -78.

[21] Zungu, L. I. 2016. Mine health and safety council: Guidelines for the South African small-scale mining to comply with the mine health and safety act. University of South Africa, MHSC/061/1415.

[22] Lim, M., Murray, J., Dowdeswell, R., Glynn, J. and Sonnenberg, P. (2011). Unnatural deaths in South African platinum miners, 1992-2008. PloS One, 6(9), e22807

[23] Green, J., Bosscha, P., Candy, L., Hlophe, K., Coetzee, S. and Brink, S. (2010). Can a robot improve mine safety? In: Proceedings of the $25^{\text {th }}$ International Conference of CAD/CAM, Robotics \& Factories of the future, Conference (2010).

[24] Zikmund, W., Babin, B., Carr, J. \& Griffin, M. (2012). Business research methods, Boston: Cengage Learning.

[25] Collis, J. and Hussey, R. (2013). Business research: A practical guide for undergraduate and postgraduate students, Basingstoke, Hampshire: Palgrave Macmillan.

[26] Burns, R. and Burns, R. (2008). Business research methods and statistics using SPSS. London: Sage.

[27] Bryman, A. and Bell, E. (2015). Business research methods. Oxford: Oxford University Press, USA.

[28] Cooper, D. and Schindler, P. (2013). Business research methods. New York: Prentice Hall Publishers.

[29] Kothari, C. (2014). Research methodology: Methods and techniques, Delhi: New Age International. 